\title{
Avaliabilidade da Política de Práticas Integrativas e Complementares do município de Recife-PE
}

Evaluation of the Policy of Integrative and Complementary Practices of Recife-PE Municipality Evaluabilidad de PNPIC Política de la municipalidad de Recife-PE

\section{Gisele Cazarin ${ }^{1}$; Sebastianjorge Florêncio Ferreira de Lima ${ }^{2}$; Iracema de Almeida Benevides ${ }^{3}$}

\section{Resumo}

As práticas integrativas e complementares (PICS) vêm ganhando destaque na saúde pública, configurando-se como escolhas promissoras na integralidade do cuidado. O município do RecifePE adotou, em 2004, política própria de práticas integrativas e complementares (PMPIC). Estudos de avaliabilidade são importantes ferramentas de apoio às decisões, e na implementação de políticas e ações, contribuindo para sua qualificação. Objetiva-se apresentar os resultados da avaliabilidade da PMPIC, a partir da elaboração e validação do seu modelo téorico lógico (ML). Os dados foram coletados a partir de entrevistas com seis informantes-chave, no período de julho a outubro de 2016. Além de revisão de documentos oficiais e técnicos. Procedeu-se a análise de conteúdo temático das entrevistas com agrupamento conforme temas afins. O estudo foi aprovado pelo Comitê de Ética em Pesquisa do Instituto de Medicina Integral Prof. Fernando Figueira (IMIP). O ML compôs-se de três componentes: gestão e intersetorialidade, atenção à saúde, e educação, formação e comunicação. Para cada componente foram desenhados os recursos, as atividades e os resultados esperados, como também as relações entre eles. A análise dos dados apontou poucas modificações, indicando aproximação entre a ferramenta proposta (ML) e a PMPIC. Entre as principais contribuições temos: inserção de aplicativo informatizado que contemple a necessidade de informações da área; investimentos no financiamento tripartite, nas ações de matriciamento, na inserção da PICS como campo de práticas e no trabalho compartilhado em rede. O estudo contribuiu para melhor entendimento do programa e dos mecanismos implicados na sua operacionalização, favorecendo seu aperfeiçoamento.

Descritores: Avaliação em Saúde; Terapias Complementares; Saúde Pública; Políticas Públicas de Saúde.

\footnotetext{
Abstract

Integrative and complementary practices (PICS) have been gaining prominence in public health, configuring themselves as promising choices in the integrality of care. The municipality of RecifePE adopted, in 2004, its own policy of integrative and complementary practices (PMPIC). Evaluation studies are important tools to support decisions, and in the implementation of policies and actions, contributing to their qualification. The objective of this paper is to present the results of the PMPIC's evaluation, based on the elaboration and validation of its logical theoretical model (LTM). Data were collected from interviews with six key informants, from July to October 2016. In addition to review of official and technical documents. The thematic content analysis of the group

1 Mestre em Saúde Pública pela Fundação Oswaldo Cruz/FIOCRUZ-PE. Docente e pesquisadora do Instituto de Medicina Integral Prof. Fernando Figueira (IMIP). Rua dos Coelhos, 300, Boa Vista, Recife, PE. CEP: 50070-550. Email: gcazarin@gmail.com

${ }^{2}$ Especialista em homeopatia pela Sociedade de Homeopatia de Pernambuco. Docente da Sociedade de Homeopatia de Pernambuco.

${ }^{3}$ Mestre em Economia e Políticas de Saúde pela Universidade Bocconi, Milão, Itália. Afiliada à Associação Brasileira de Medicina Antroposófica (ABMA).
} 
ISSN 2179-6750

interviews was carried out according to related themes. The study was approved by the Research Ethics Committee of the Instituto de Medicina Integral Prof. Fernando Figueira (IMIP). LTM was composed of three components: management and intersectoriality, health care, and education, training and communication. For each component the resources, activities and expected results were designed, as well as the relationships between them. The data analysis showed few modifications, indicating an approximation between the proposed tool (LTM) and the PMPIC. Among the main contributions can be pointed: insertion of a computerized application that contemplates the need of information of the area; investment in tripartite funding, in the actions of matriciamento, in the insertion of the PICS as field of practices and in the shared work in network. The study contributed to a better understanding of the program and the mechanisms involved in its operationalization, favoring its improvement.

Key-words: Health Evaluation; Complementary Therapies; Public Health; Public Health Policy.

\section{Resumen}

Las prácticas integrativas y complementarias (PICS) vienen ganando destaque en la salud pública, configurándose como enfoques prometedores en la integralidad del cuidado. El municipio de Recife-PE ha aprobado, en 2004, una política de prácticas integrativas y complementarias para la municipalidad. Los estudios de evaluación son importantes herramientas de apoyo a las decisiones, y en la implementación de políticas y acciones, contribuyendo a su calificación. Se pretende presentar los resultados de la evaluación de la PMPIC, a partir de la elaboración y validación de su modelo téorico lógico (ML). Los datos fueron recolectados a partir de entrevistas con seis informantes clave, en el período de julio a octubre de 2016. Además de la revisión de documentos oficiales y técnicos. Se realizó el análisis de contenido temático de las entrevistas con agrupamiento conforme temas afines. El estudio fue aprobado por el Comité de Ética en Investigación del Instituto de Medicina Integral Fernando Figueira (IMIP). El ML se compuso de tres componentes: gestión e intersectorialidad, atención a la salud, y educación, formación y comunicación. Para cada componente se han diseñado los recursos, las actividades y los resultados esperados, así como las relaciones entre ellos. El análisis de los datos apuntó pocas modificaciones, indicando aproximación entre la herramienta propuesta (ML) y la PMPIC. Entre las principales contribuciones tenemos: inserción de aplicación informatizada que contemple la necesidad de informaciones del área; Inversión en la financiación tripartita, en las acciones de matriciación, en la inserción de la PICS como campo de prácticas y en el trabajo compartido en red. El estudio contribuyó a un mejor entendimiento del programa y de los mecanismos implicados en su operacionalización, favoreciendo su perfeccionamiento.

Palabras-claves: Evaluación en Salud; Terapias Complementarias; Salud Pública; Políticas Públicas de Salud.

\section{Introdução}

No Brasil, crescente tem sido o interesse na valorização e ampliação do acesso às práticas terapêuticas, denominadas de medicina alternativa e complementar/medicina tradicional (MAC/MT), ou práticas integrativas e complementares (PICS), nomenclatura adotada pelo Ministério da Saúde ${ }^{1}$. Sua implementação, no campo das políticas públicas nacionais, ganhou impulso a partir da década de 1980, principalmente, pela acupuntura, cujo processo de legitimação e legalização liderou esse percurso no país ${ }^{2,3,4}$. 
ISSN 2179-6750

Esse movimento esteve alinhado ao cenário internacional, onde reconhecidos Órgãos Internacionais, a exemplo da Organização Mundial de Saúde (OMS) ratificaram, por meio de Conferências Internacionais, a necessidade de integração entre medicinas tradicionais e práticas terapêuticas 'não convencionais' nos serviços públicos de saúde. Essa preocupação foi enfatizada, anos antes, na Conferência de Alma Ata (1978) que preconizou tornar acessíveis as medicinas 'nativas', integrando-as ao sistema oficial ${ }^{2}$.

O expressivo uso dessas práticas, pela classe média brasileira, aumentou a influência social pela sua institucionalização, onde a publicação do documento final da VIII Conferência Nacional de Saúde teve importante papel ao recomendar a introdução de práticas alternativas de assistência à saúde no âmbito dos serviços de saúde ${ }^{2}$.

O ápice do reconhecimento ocorreu anos mais tarde, com a publicação, em 2006, da Política Nacional de Práticas Integrativas e Complementares (PNPIC) ${ }^{1}$. A PNPIC indica que essas ações sejam realizadas a partir da rede de atenção, com ênfase na atenção primária à saúde (APS). A PNPIC contempla tanto sistemas médicos complexos ${ }^{2}$, a exemplo da medicina tradicional chinesa (MTC), homeopatia, medicina antroposófica, quanto a utilização de recursos terapêuticos ${ }^{4}$, como: utilização de plantas medicinais e fitoterapia e termalismo/crenoterapia. O escopo da PNPIC foi recentemente ampliado sendo incluída a oferta das seguintes práticas: arteterapia, meditação, musicoterapia, tratamento naturopático, tratamento osteopático, tratamento quiroprático e reiki ${ }^{5}$.

Em que pese os avanços no reconhecimento, por meio da ampliação das práticas a serem ofertadas nos serviços públicos de saúde, ainda persistem problemas quanto à definição do que se compreende como PICS dentro da PNPIC, o que traz uma dificuldade e um desafio à inserção dessas práticas nos serviços públicos de saúde, em especial no seu lócus preferencial: a atenção primária à saúde ${ }^{6,7,8}$. Outro aspecto relevante diz respeito à qualidade da informação referente ao registro da produção desse tipo de atendimento nos Sistemas de informação oficiais (Sistema de Informação Ambulatorial - SIA/SUS e Cadastro Nacional de Estabelecimentos de Saúde - CNES). Onde há limitações importantes pela não permissão do registro adequado dessas práticas, o que compromete sua mensuração, monitoramento e avaliação ${ }^{6}$.

Ademais, atualmente, ainda que, esforços tenham sido envidados na institucionalização dessas práticas, há necessidade de investimentos na ampliação da oferta e implementação desses serviços no Sistema Único de Saúde (SUS) ${ }^{3}$. Para Santos; Tesser ${ }^{9}$, apesar do acesso às PICS ser ainda restrito, houve aumento da procura social por essas práticas mediante méritos próprios, tais como: reposicionamento do usuário como centro do paradigma médico; consideração da relação curador (usuário como elemento fundamental da terapêutica); busca de meios terapêuticos simples 
ISSN 2179-6750

(menos dependentes de tecnologia científica 'dura', menos caros), entretanto, com igual ou maior eficácia nas situações comuns de adoecimento; e estímulo à construção de uma medicina que busca acentuar a autonomia do usuário, tendo como categoria central a saúde e não a doença.

O município do Recife (Pernambuco) idealizou e implantou, em 2004, política de Práticas Integrativas e Complementares (PMPIC), instituída oficialmente em 2012, por meio de Portaria municipal ${ }^{10}$. A PMPIC conta com duas Unidades de Cuidado Integrais a Saúde (UCIS) que devem funcionar como referência técnica para a rede de atenção à saúde municipal saúde. E seis Unidades de Apoio a Práticas Integrativas (NAPI), distribuídas nos diversos Distritos Sanitários (DS), cuja atuação deve se dar de forma conjunta com as equipes de APS, à luz do apoio matricial; nos moldes do trabalho preconizado pelo Núcleo de Apoio ao Saúde da Família (NASF). Cabe ressaltar que os NAPI, caracterizam-se como equipamentos de saúde peculiares à PMPIC do referido município. Entre as principais práticas realizadas no âmbito da PMPIC estão: MTC (acupuntura, Tai Chi Chuan, Lian Gong), homeopatia, medicina ayurvédica, yoga, bioenergética, psicomotricidade relacional, automassagem, e fitoterapia ${ }^{10}$.

Considerando a relevância das intervenções de APS na consolidação dos sistemas de saúde, a avaliação e o monitoramento constituem-se como ferramentas potenciais de apoio às decisões, no planejamento e implementação dessas políticas e ações contribuindo na sua qualificação. $O$ interesse em avaliar intervenções vem crescendo, nos últimos anos, em consequência da ampliação da oferta dos serviços de saúde, bem como, para responder às novas necessidades e problemas de saúde ${ }^{11}$.

No que se refere às políticas direcionadas às PICS reconhece-se que, apesar dos esforços, há escassez tanto na definição de instrumentos, indicadores e fluxos para monitoramento e avaliação, quanto escassez de estudos avaliativos sobre a inserção dessas práticas nos serviços de saúde ${ }^{6,8}$. Considerando tal lacuna, os estudos de avaliabilidade têm sido muito úteis.

A avaliabilidade refere-se a uma apreciação prévia acerca das possibilidades de avaliação de uma intervenção. $\mathrm{O}$ emprego dessa metodologia permite maior entendimento entre os interessados na avaliação, sendo apropriado na (re) definição de objetivos e metas e indicando áreas prioritárias para futuras avaliações ${ }^{12}$.

\section{Métodos}

Foi realizado um estudo de avaliabilidade ou avaliação exploratória. Trata-se de um método analítico que permite identificar se o estágio de desenvolvimento da intervenção favorece sua avaliação, e se seus objetivos respondem à situação-problema que a originou. Não se presta a 
ISSN 2179-6750

mostrar se a intervenção pode ser avaliada (qualquer intervenção pode ser avaliada), mas se a avaliação é capaz de contribuir para melhorar sua performance ${ }^{13}$.

A estratégia de pesquisa utilizada foi o estudo de caso único, tendo a Política de Práticas Integrativas do município do Recife como unidade de análise. $\mathrm{O}$ caso foi definido de acordo com as recomendações preconizadas por Yin ${ }^{14}$ na condução de um de estudo de caso único. Para o mencionado autor, esse tipo de estudo se justifica pela caracterização do caso como peculiar, onde o desenho de casos múltiplos ou uma amostra estatisticamente representativa de casos seria inviável.

O município estudado (Recife) é capital do Estado de Pernambuco, e do ponto de vista administrativo divide-se em seis Regiões Político-Administrativas (RPA) e oito Distritos Sanitários (DS). O Índice de Desenvolvimento Humano (IDH) (0,772: classificado na faixa de alto desenvolvimento humano) é o melhor das capitais nordestinas, ocupando, em 2010, a primeira colocação entre os municípios pernambucanos para esse indicador. Em contraste com o valor alcançado para o índice que afere a desigualdade na distribuição de renda (índice de Gini: 0,49), considerado o mais alto do estado ${ }^{15}$.

As etapas do estudo foram adaptadas de Leviton ${ }^{16}$, a saber: 1. revisão de documentos para esclarecimento sobre os objetivos e metas do programa; 2. modelização da intervenção (desenho do modelo teórico lógico-ML), 3. elaboração/validação do ML, e 4. elaboração de recomendações. O ML é um instrumento bastante utilizado em avaliação, uma vez que comunica a operacionalização da intervenção evidenciando seus produtos e resultados esperados ${ }^{17}$.

Para construção do ML preliminar foram identificados e analisados alguns documentos oficiais e técnicos da política, posteriormente, com a ajuda dos informantes-chave novos documentos foram inseridos no rol.

Os documentos analisados foram: Portaria $\mathrm{n}^{\circ}$ 122, de 6 de julho de 2012 (que dispõe a criação da política municipal de práticas integrativas e complementares), Portaria GM nº 971, de 03 de maio de 2006 (que institui a Política Nacional de Práticas Integrativas e Complementares), Planos Municipais de Saúde 2010-2013 e 2014-2017, Planos Municipais de Saúde e Documentos Técnicos institucionais da Gerência de Atenção Básica da Secretaria de Saúde do município.

O ML preliminar foi disposto num diagrama contendo: recursos, componentes, atividades, resultados intermediários e resultado final, como também as relações entre eles possibilitando a análise da organização, dos objetivos, e dos resultados esperados. Posteriormente, esse foi submetido aos informantes-chave, por meio de entrevistas semiestruturadas. Os entrevistados foram eleitos de forma intencional, de acordo com sua experiência/expertise em atividades de pesquisa, ensino, gestão, atuação profissional na política, como também indicação por outro informante- 
ISSN 2179-6750

chave. Dessa forma, além da coordenação geral da PMPIC foram entrevistados, a assessoria da PMPIC, Gerência da UCIS Guilherme Abath, dois profissionais integrantes da UCIS e representante de instituição local de ensino e pesquisa, totalizando seis entrevistados.

Para análise do ML preliminar os entrevistados deveriam considerar os seguintes critérios: clareza na descrição dos objetivos da PMPIC; suficiência/adequação dos elementos do ML e suas relações, plausibilidade e lógica das relações propostas.

As entrevistas, com duração média de uma hora e meia, foram conduzidas pelo pesquisador principal, mediante consentimento livre e esclarecido dos participantes. O registro das entrevistas foi realizado por gravação em meio digital e anotações manuscritas. As gravações foram transcritas integralmente por um profissional independente. As entrevistas foram realizadas no período de período de julho a outubro de 2016.

Para definição do número suficiente de entrevistados foi utilizado o critério de saturação teórica. Onde se levou em conta tanto a reincidência de ideias e não surgimento de novos elementos, como também a complementaridade das informações coletadas ${ }^{18,19}$.

Procedeu-se a análise de conteúdo temático mediante leitura minuciosa e repetida do material com o objetivo de compreensão e agrupamento em categorias relevantes conforme temas afins. Os trechos das entrevistas foram organizados em quadros temáticos, tal agrupamento possibilitou a identificação de temas recorrentes, os quais foram divididos em recursos, atividades e resultados. A partir disso, o ML foi reformulado.

O estudo foi aprovado pelo Comitê de Ética em Pesquisa do Instituto de Medicina Integral Prof. Fernando Figueira (IMIP), como parte integrante da pesquisa Avaliabilidade da Política de Homeopatia no Município do Recife-PE, sob parecer nº 1.673.059/2016.

\section{Resultados}

A revisão dos documentos mostrou-se apropriada para fins da delimitação do objetivo da PMPIC que é ${ }^{20}$ :

Democratizar e implementar a Política Municipal de Práticas Integrativas e Complementares, ampliando o acesso dos usuários às práticas integrativas, terapias vibracionais e complementares, para melhorar a qualidade de vida, o bem estar e a longevidade do ser/cidadão de forma segura, descentralizada, articulada intersetorialmente, hierarquizada e humanizada, em cogestão com os trabalhadores, movimentos sociais e comunitários (p. 61).

As metas do programa estão voltadas principalmente para a expansão do acesso, por meio de implementação de linha de cuidado integral, tendo a APS como ordenadora. Entre as metas principais temos: ampliação da oferta de procedimentos de PIC nas Unidades de Saúde da Família e 208 
ISSN 2179-6750

no NAPI, e investimento da formação permanente em serviço sobre tais práticas ${ }^{20,21}$.

$\mathrm{O}$ resultado final do ML, inicialmente proposto, derivou do objetivo geral do programa explicitado acima, sendo definido, provisoriamente como: ampliação do acesso aos cuidados integrais no município do Recife. Tal resultado, na opinião dos entrevistados, não refletia os propósitos da PMPIC. A maioria dos informantes-chave concordou que ele seria mais bem definido como: aumento da oferta e qualificação dos cuidados integrais no município do Recife (Figura 1). Já que acesso, como constava anteriormente, depende de questões mais amplas, como a ampliação da cobertura, ainda restrita, além de investimento na divulgação a respeito de tais práticas tanto para profissionais da rede, quanto para usuários.

A PMPIC nasceu num contexto onde o modelo de atenção à saúde em Defesa da Vida, baseado no método Paideia, proposto por Campos; Domitti ${ }^{22}$, era expressivo no discurso e nas práticas municipais ${ }^{23,24}$. Dessa forma, mesmo com o arrefecimento posterior das discussões sobre os pilares do referido método (cogestão de coletivos, clínica ampliada, acolhimento, projetos terapêuticos singulares- PTS e apoio matricial), muitos desses encontraram-se presentes nas estratégias propostas pela política, tendo sido, portanto incorporados no desenho do ML. Para cada componente foram elaborados e validados a estrutura necessária (recursos) e as atividades a serem executadas para se atingir os objetivos e os resultados esperados.

De um modo geral, a maior parte dos entrevistados avaliou positivamente a ferramenta proposta para avaliabilidade (ML), uma vez que poucas foram as inclusões/reformulações sugeridas. Houve consenso a respeito dos elementos do modelo (componentes, atividades, estrutura e principais resultados) e das relações entre eles. O ML da PMPIC (Figura 1) ficou composto por três componentes: 1. gestão e intersetorialidade; 2. atenção à saúde, e 3. educação, formação e comunicação.

Quanto aos aspectos de estrutura/recursos, a discussão voltou-se para a inserção do sistema e-SUS, em substituição ao SIA-SUS, como sistema de aferição da produção relativa a PIC. Na opinião dos entrevistados, a implantação do novo sistema (e-SUS) representou um avanço, no entanto, ainda insuficiente/inadequado às necessidades de informação da área técnica de PIC. Uma vez que não apresenta a especificidade requerida ao processo de tomada de decisão, além de estimular a lógica de produção quantitativa. Sugeriu-se que no ML fosse inserido o desenvolvimento de aplicativo informatizado que abrangesse as informações necessárias ao monitoramento sistemático da política. 
ISSN 2179-6750

Figura 1. Modelo teórico lógico da Política Municipal de Práticas Integrativas em Complementares do município do Recife-PE, 2017.

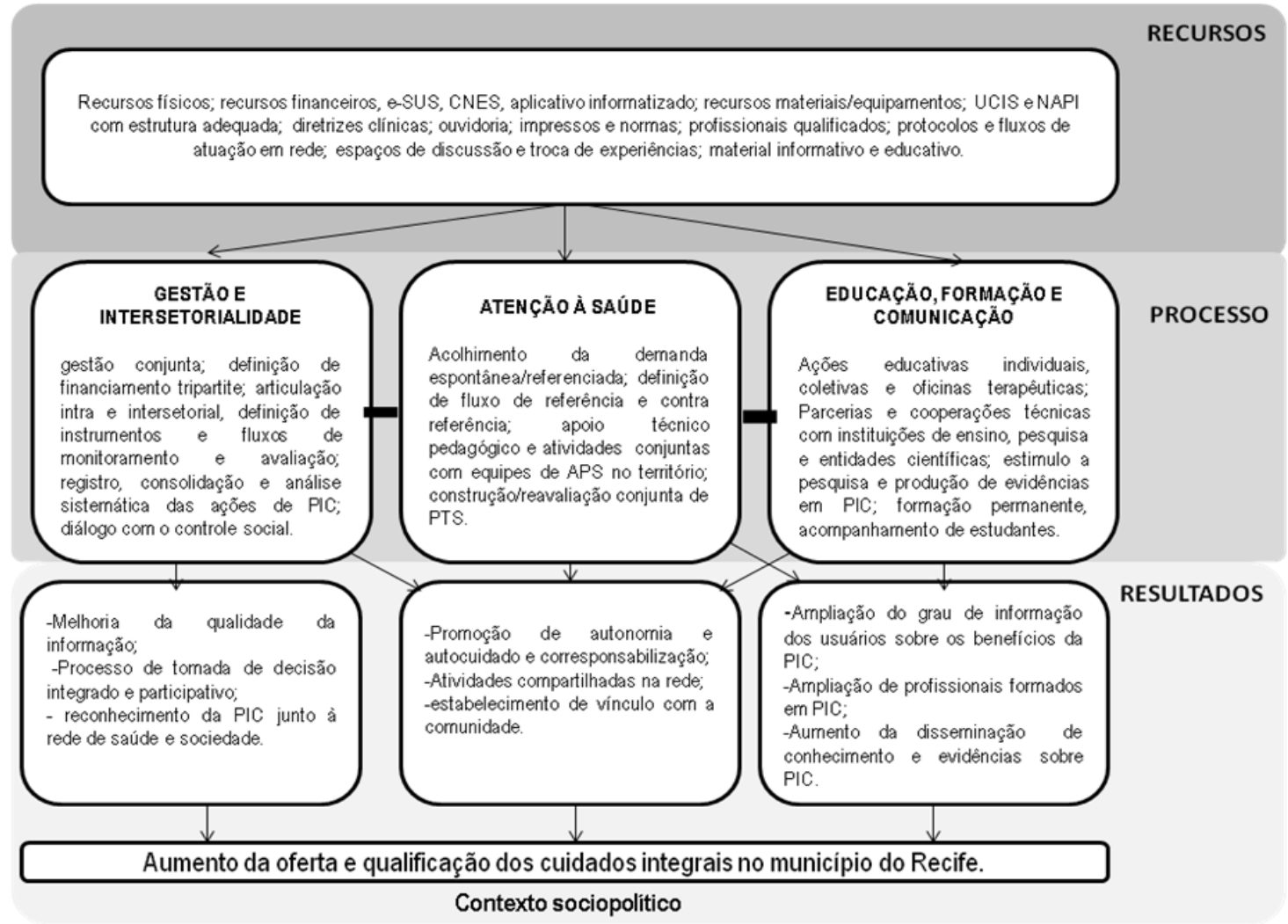

Fonte: Elaboração própria (2017).

No que se refere ao processo, houve entendimento de que algumas práticas, a exemplo da acupuntura, da homeopatia e da medicina antroposófica, atualmente, não têm condições de serem matriciadas. Alguns entrevistados chamaram atenção de que o apoio técnico pedagógico apresenta dificuldades na sua implementação devido à expertise exigida à aplicação dessas especialidades (na maior parte das vezes, exercida por profissionais médicos). Soma-se a isso a escassez de oferta de formação específica nessas áreas, assim como o fato do município contar com poucos profissionais especialistas. Ponderou-se que o tempo despendido à realização de consultas nessas áreas (superior a uma consulta comum) as caracterizam, no momento, como especialidades a serem exercidas a nível ambulatorial, ou seja, nas UCIS e não nos NAPI.

A restrição de profissionais disponíveis em algumas especialidades refletiu no componente do ML de educação, comunicação e mobilização, uma vez que alguns serviços não podem, atualmente, serem disponibilizados como campo de práticas. Agravado pelos equipamentos de PIC municipais apresentarem heterogeneidade nas práticas ofertadas, o que dificulta a inserção de estudantes exclusivos.

$$
210
$$


ISSN 2179-6750

Também mereceu destaque nas falas, a dificuldade de articulação intrassetorial das PIC (com os outros equipamentos municipais de saúde). Nesse caso, há pouca aceitação e precária comunicação, o que vem dificultando a realização de atividades compartilhadas em rede. Outro fator importante refere-se ao financiamento, onde foi apontada preocupação com a não definição de recursos financeiros tripartite, o que na opinião dos entrevistados pode vir a comprometer em longo prazo, a sustentabilidade da política.

\section{Discussão}

Os resultados do presente estudo revelaram que a Política de Práticas Integrativas do município do Recife (PMPIC) encontra-se suficientemente estruturada em termos dos seus componentes e resultados esperados.

O estudo de avaliabilidade da PMPIC contribuiu para melhor entendimento a respeito da imagem-objetivo do programa, a qual aparentemente não estava clara para os envolvidos. A revisão dos documentos apontou para ampliação do acesso às PICS como objetivo geral, mas como tal conceito, na visão dos entrevistados, seria abrangente, assim como para alcançá-lo seria necessário investimento anterior na ampliação da cobertura referente às mencionadas práticas, optou-se pela revisão do objetivo, no sentido de ampliação da oferta.

O ML explicitou os mecanismos implicados na operacionalização da PMPIC no âmbito municipal de modo a alcançar o aumento da sua oferta, enquanto opção de cuidado dentro do arsenal terapêutico voltado à integralidade da atenção. Isso foi particularmente útil na explicação das relações entre a intervenção, sua implementação e seus resultados esperados ${ }^{25}$. Nesse caso, verificou-se coerência na relação entre o problema (baixa oferta de PICS no município) e a intervenção desenhada para atendê-lo (PMPIC).

A pouca clareza com que os programas e políticas de saúde apresentam seus objetivos e a falta de consenso, entre os envolvidos, sobre suas atividades e seus resultados esperados são queixas frequentes nos serviços de saúde. Nesse sentido, o desenho do ML possibilita uma visão mais clara acerca da racionalidade da intervenção, servindo de ferramenta de comunicação entre os envolvidos. Ademais, sua construção elucida as coerências internas do programa, contribuindo na identificação de lacunas e resultados fora da realidade ou incoerentes ${ }^{17,26}$.

Foram apontados aspectos da política não pertinentes de serem avaliados na sua conjuntura atual, a exemplo dos sistemas de informação não adaptados à realidade das PICS, dificuldades na definição de recursos financeiros tripartite, no matriciamento e no acompanhamento de estudantes para alguns tipos de práticas, além de dificuldades no desenvolvimento de trabalho compartilhado 
ISSN 2179-6750

em rede. Entretanto, a maior parte dos entrevistados optou por mantê-los no ML, pelo entendimento de que ele é uma representação de como o programa supostamente deveria acontecer no plano ideal, não considerando as possíveis barreiras para sua execução ${ }^{26}$. O processo de desenho do ML estimulou os participantes da pesquisa, integrantes da política, a procurarem formas de fortalecer o papel da PICS no âmbito rede de saúde, com ênfase na APS, tendo sido também útil na identificação de oportunidades de melhoria.

\section{Conclusão}

Estudos de avaliabilidade apresentam como principais finalidades proporcionar um ambiente favorável para a avaliação, construir entendimentos entre os envolvidos sobre as características, os objetivos, as expectativas e as necessidades de informação sobre uma intervenção/programa. Além de buscar o consenso quanto ao interesse na realização da avaliação, maximizando sua potencialidade, sua oportunidade e sua utilidade ${ }^{16}$.

Os resultados do presente estudo apontam que o ML proposto para a PMPIC constitui-se num instrumento importante na realização de investigação e discussão sobre a referida política, assim como realização de futuras avaliações a seu respeito. Conclui-se que o estudo contribuiu para melhor entendimento do programa e dos mecanismos implicados na sua operacionalização, favorecendo seu aperfeiçoamento.

\section{Agradecimentos}

Os autores agradecem a equipe da Secretaria Municipal de Saúde do Recife pela colaboração na concepção desse artigo.

\section{Referências}

1. Brasil, Ministério da Saúde, Secretaria de Atenção à Saúde, Departamento de Atenção Básica. Política Nacional de Práticas Integrativas e Complementares no SUS - PNPICSUS. Brasília, DF: Ministério da Saúde; 2006. 92 p. (Série B. Textos Básicos de Saúde).

2. Luz TM. Questões e desafios colocados para o estudo das racionalidades médicas e das práticas de saúde na atualidade: ensino, pesquisa e exercício da atenção profissional. In: Pinheiro R, Silva Junior AG, organizadores. Cidadania no cuidado: o universal e o comum na integralidade das ações em saúde. Rio de Janeiro: IMS/UERJ-CEPESC; 2011. p. 295-305. 
ISSN 2179-6750

3. Lacerda A. Homeopatia popular e praticada por médicos: desafios da integração das práticas no contexto da promoção da saúde. In: Pinheiro R, Silva Junior AG, organizadores. Cidadania no cuidado: o universal e o comum na integralidade das ações em saúde. Rio de Janeiro: IMS/UERJ-CEPESC; 2011. p. 305-17.

4. Nascimento MC, Barros NF, Nogueira MI, Luz MT. A categoria racionalidade médica e uma nova epistemologia em saúde. Cien Saude Colet. 2013;18(12):3595-604. http://dx.doi.org/10.1590/S1413-81232013001200016.

5. Brasil. Ministério da Saúde. Portaria $n^{\circ} 145$ de 11 de janeiro de 2017: altera procedimentos na Tabela de Procedimentos, Medicamentos, Órteses, Próteses e Materiais Especiais do SUS para atendimento na Atenção Básica. Diário Oficial da União. 2017 jan 13;(10):32-34.

6. Sousa IMC, Bodstein RCA, Tesser CD, Santos FAS, Hortale VA. Práticas integrativas e complementares: oferta e produção de atendimentos no SUS e em municípios selecionados. Cad Saude Publica. 2012 Nov;8(11):2143-54. http://dx.doi.org/10.1590/S0102311X2012001100014.

7. Lima KMSV, Silva KL, Tesser CD. Práticas integrativas e complementares e relação com promoção da saúde: experiência de um serviço municipal de saúde. Interface (Botucatu). 2014;18(49):261-72. http://dx.doi.org/10.1590/1807-57622013.0133.

8. Sousa IMC, Tesser CD. Medicina Tradicional e Complementar no Brasil: inserção no Sistema Único de Saúde e integração com a atenção primária. Cad Saude Publica. 2017 Jan;33(1):115. http://dx.doi.org/10.1590/0102-311x00150215.

9. Santos MC, Tesser CD. Um método para a implantação e promoção de acesso às Práticas Integrativas e Complementares na Atenção Primária à Saúde. Cienc Saude Colet. 2012;17(11):3011-24. http://dx.doi.org/10.1590/S1413-81232012001100018.

10. Recife. Secretaria Municipal de Saúde. Portaria $n^{\circ}$ 122, de 6 de julho de 2012. Criação da Política Municipal de Práticas Integrativas e Complementares. Diário Oficial do Município de Recife. 2012 set 13:1-3.

11. Figueiró AC, Frias PG, Navarro LM. Avaliação em saúde: conceitos básicos para a prática nas instituições. In: Samico I, Felisberto E, Figueiró AC, Frias PG, organizadores. Avaliação em Saúde: bases conceituais e operacionais. Rio de Janeiro: MedBook; 2010. p. 1-14.

12. Bezerra LCA, Alves CKA, Reis YAC, Samico I, Felisberto E, Carvalho ALB, Silva GS. Identificação e caracterização dos elementos constituintes de uma intervenção: pré-avaliação da política ParticipaSUS. Cien Saude Colet. 2012 Abr;17(4):883-900. http://dx.doi.org/10.1590/S1413-81232012000400011. 
ISSN 2179-6750

13. Thurston WE, Ramaliu A. Evaluability assessment of a survivors of torture program: lessons learned. Can J Program Eval. 2005;20(2):1-25.

14. Yin RK, organizador. Estudo de caso: planejamento e métodos. Porto Alegre: Bookman; 2005. Yin RK. Projeto dos estudos de caso: identificação e estabelecimento da lógica do seu estudo de caso. p. 29-72.

15. Instituto Brasileiro de Geografia e Estatística [Internet]. [Brasília]: IBGE; [date unknown acesso em 2016 mar 21]. Disponível em: https://cidades.ibge.gov.br/brasil/pe/recife/panorama

16. Leviton LC, Khan LK, Rog D, Dawkins N, Cotton D. Evaluability Assessment to Improve Public Health Policies, Programs, and Practices. Annu Rev Public Health. 2010 Apr 21;31:213-33. https://doi.org/10.1146/annurev.publhealth.012809.103625.

17. Champagne F, Brouselle A, Hartz Z, Contandriopoulos A-P. Modelizar as Intervenções. In: Brousselle A, Champagne F, Contandriopoulos, A-P, Hartz Z, organizadores. Avaliação: conceitos e métodos. Rio de Janeiro: Fiocruz; 2011. p. 61-74.

18. Gomes, R, Souza ER, Minayo, MCS, Malaquias JV, Silva CFR. Organização, processamento, análise e interpretação de dados: o desafio da triangulação. In: Minayo MCS, Assis SG, Souza ER, organizadores. Avaliação por triangulação de métodos: abordagem de programas sociais. Rio de Janeiro: Fiocruz; 2005. p. 185-223.

19. Fontanella BJB, Ricas J, Turato ER. Amostragem por saturação em pesquisas qualitativas em saúde: contribuições teóricas. Cad Saude Publica. 2008 Jan;24(1):17-27.

20. Recife, Secretaria Municipal de Saúde. Plano Municipal de Saúde 2010-2013. Recife: Secretaria de Saúde; 2010 [acesso 2017 fev 10]. Disponível em: http://www2.recife.pe.gov.br/wp-content/uploads/PMS-2010-2013.pdf

21. Recife, Secretaria Municipal de Saúde. Plano Municipal de Saúde 2014-2017. Recife: Secretaria de Saúde, 2014 [acesso 2017 fev 10]. Disponível em: http://www2.recife.pe.gov.br/sites/default/files/plano_municipal_de_saude_2015_revisado_m enor.pdf

22. Campos GWS, Domitti AC. Apoio Matricial e equipe de referência: Uma metodologia para gestão do trabalho interdisciplinar em saúde. Cad Saude Publica. 2007 Fev;23(2):399-407. http://dx.doi.org/10.1590/S0102-311X2007000200016.

23. Alves CKA, Couto G, Coêlho BP, Feitosa T, Cazarin G, Coelho Neto G, et al. Redes cotidianas de cuidado: desafios e possibilidades de incorporação de redes sociais e ação política na produção de cuidado em saúde. In: Pinheiro R, Silva Junior AG, organizadores. Cidadania do cuidado. Rio de Janeiro: CEPESC/ABRASCO; 2011. p.125-49. 
ISSN 2179-6750

24. Almeida RCC. Práticas integrativas e complementares e o modelo de defesa da vida: análise das novas políticas do SUS no Recife no período de 2009 a 2011 [dissertação]. Recife, PE: Universidade Federal de Pernambuco; 2012.

25. Chen HT. Practical program evaluation: assessing and improving planning, implementation, and effectiveness. Thousand Oaks, CA: SAGE Publications; 2004. 304 p.

26. Bezerra LCA, Cazarin G, Alves CKA. Modelagem de programas: da teoria à operacionalização. In: Samico I, Felisberto E, Figueiró AC, Frias PG, organizadores. Avaliação em Saúde: bases conceituais e operacionais. Rio de Janeiro: MedBook; 2010. p. $65-78$. 